\title{
Effects of Multiplicative Colored Noise on Bacteria Growth
}

\author{
Hong-Yan Liao, Bao-Quan Ai, and Lian Hu \\ School of Physics \& Telecommunication Engineering, South China Normal University, GuangZhou, People's Republic of China
}

Received on 12 April, 2007

\begin{abstract}
We use the logistic growth model to describe the bacterium growth in the presence of a Gaussian colored noise. The effects of multiplicative colored noise on the steady state probability distribution of the bacterium growth were investigated. Our results show that increasing the strength of the multiplicative colored noise may lead to decreasing bacterium number and even bacterium extinction. Our studies also indicate that increasing the correlate time of the noise may facilitate the bacterium growth to some extent.
\end{abstract}

Keywords: Colored noise; Bacteria growth; Steady probability distribution

\section{INTRODUCTION}

Recently, nonlinear stochastic systems with noise terms have attracted extensive investigations and the concept of noise-induced transition has wide applications in some fields, such as physics, chemistry, and biology [1-4]. Usually, in these systems, the noise affects the dynamics through a system variable, i.e., the noise is both multiplicative and additive [5]. The focal theme of these investigations is to study the steady state properties of systems in which fluctuations, generally applied from outside, are considered independent of the system's characteristic dissipation. In a previous work [6], we studied the steady state properties of the logistic growth model in the presence of the correlated white noises. However, in some cases, the noise may be colored, for example, Masoliver and co-workers [7] investigated the mean first-passage time for a bistable system driven by Gaussian colored noise.

There are several mathematical functions for describing microbial growth curves, such as three-phase linear, logistic, Gompertz, Von Bertalanffy, Richards, Morgan, Weibull, France and Baranyi function [8]. A new logistic model for bacterial growth successfully described sigmoidal growth curves of Escherichia coli at various initial cell concentrations and constant temperatures [9]. The model predicted well the bacterial growth curves, similar to the Baranyi model and better than the modified Gompertz model [9]. The improved model has the potential to successfully predict microbial growth [10].

In this paper, we study the bacterial growth in the presence of a colored noise. We emphasize on finding how the colored noise effects the bacterial growth.

\section{THE BACTERIUM GROWTH MODEL}

The logistic growth model has been used in many cases as a basic model of both cell growth and, more particularly, tumor cell growth [11]. In this paper, we consider bacterium growth. The logistic differential equation is

$$
\frac{d x}{d t}=a x-b x^{2},
$$

where $x$ is the bacterium number, $a$ the growth rate, and $b$ the decay rate. We consider effects due to some external factors such as temperature, $\mathrm{pH}$, energy, water activity, etc. These factors can influence the bacterium number directly as well as alter the bacterium growth and decay rate. In other words, the fluctuation of these factors affects the parameter $a$ and $b$, generating multiplicative colored noise. Providing the bacterium growth rate fluctuates, we obtain

$$
\frac{d x}{d t}=a x-b x^{2}+x Q(t)
$$

where $Q(t)$ is a Gaussian colored noise with the following properties:

$$
\langle Q(t)\rangle=0,
$$

$$
\left\langle Q(t) Q\left(t^{\prime}\right)\right\rangle=\frac{\alpha}{\tau} e^{-\frac{\left|t-t^{\prime}\right|}{\tau}},
$$

where $\alpha$ is the strength of the noise, $\tau$ is the correlation time of the noise.

In the other case, when the bacterium decay rate fluctuates, we obtain

$$
\frac{d x}{d t}=a x-b x^{2}-x^{2} H(t)
$$

where $H(t)$ is a Gaussian colored noise with the following properties:

$$
\langle H(t)\rangle=0,
$$

$$
\left\langle H(t) H\left(t^{\prime}\right)\right\rangle=\frac{D}{\tau} e^{-\frac{\left|t-t^{\prime}\right|}{\tau}},
$$

where $D$ is the strength of the noise, $\tau$ the correlation time of it. 


\section{STEADY STATE ANALYSIS AND RESULTS}

Since the bacterium number $x$ can not be negative, based on the method in $[12,13]$ we can obtain the Fokker-Planck equation for the evolution of steady probability distribution function (SPDF) corresponding to Eq. (2) under the constraint $x \geq 0$. The equation is $[12,13]$

$$
\frac{\partial P(x, t)}{\partial t}=-\frac{\partial}{\partial x} A(x, \tau) P(x, t)+\frac{\partial^{2}}{\partial x^{2}} B(x, \tau) P(x, t),
$$

where $P(x, t)$ is the probability density and

$$
\begin{gathered}
A(x, \tau)=\frac{a x-b x^{2}}{1+b \tau x}+\frac{\alpha x}{(1+b \tau x)^{2}}-\frac{\alpha b \tau x^{2}}{(1+b \tau x)^{3}}, \\
B(x, \tau)=\frac{\alpha x^{2}}{(1+b \tau x)^{2}} .
\end{gathered}
$$

The stationary probability distribution of equation is given as $[12,13]$

$$
P_{s t}(x)=\frac{N}{B(x, \tau)} \exp \left[\int^{x} \frac{A\left(x^{\prime}, \tau\right)}{B\left(x^{\prime}, \tau\right)} d x^{\prime}\right],
$$

where $N$ is a normalization constant. Using the explicit forms of $A(x, \tau)$ and $B(x, \tau)$ we obtain the following SPDF [12,13]:

$$
P_{s t}(x)=\frac{N(1+b \tau x)}{\alpha} x^{(a / \alpha-1)} \exp \left[\frac{(-b \tau x+2 a \tau-2) b x}{2 \alpha}\right] .
$$

In Figs. 1 and 2, we show the effects of the strength of the multiplicative colored noise $Q(t)$ on the steady state probability distribution (SPD). Clearly, as $\alpha$ is increased, the position of the extremum of SPD moves from a large value of $x$ to a small value of $x$, showing that the multiplicative noise is a drift term, which denotes that the multiplicative noise can push the system bacterium towards extinction [6]. In addition, the maximum value of $P_{s t}(x)$ decreases with increasing $\alpha$. These indicate that the fluctuation of bacterium growth rate $a$ can hinder the bacterium growth. The results demonstrate that the evolution of the nonlinear biological systems is suppressed by the strength of the multiplicative colored noise.

Figures 3 and 4 show the effects of the correlation time of multiplicative colored noise $Q(t)$ on the SPDF. We can see that as $\tau=0$, the colored noise becomes white noise, and $P_{s t}(x)$ reaches a maximum value at $x=7$. The maximum value of $P_{s t}(x)$ increases as $\tau$ is increased. These indicate that the large correlation time of the colored noise can advance the bacterium growth and make the evolution of bacterium more steadily. So we can abate the effects of the multiplicative noise on the nonlinear biological systems by increasing the correlation time of the noise.

In the other case, when the bacterium decay rate fluctuates, the FP equation for the evolution of the SPDF corresponding to Equation (5) is the same as Equation (8) and here

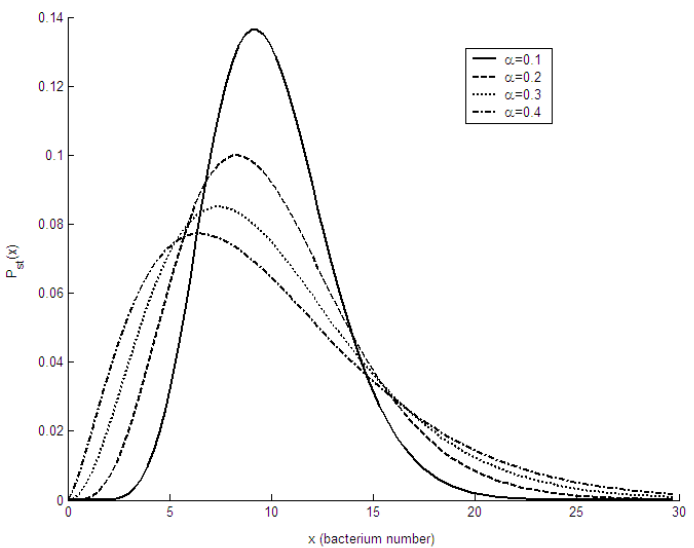

FIG. 1: Plot of $P_{s t}(x)$ (probability density) vs. $x$ (bacterium number) for low values of the noise intensity $a . a=1, b=0.1, \tau=0.1$ and $\alpha=0.1,0.2,0.3$ and 0.4 , respectively(units are arbitrary).

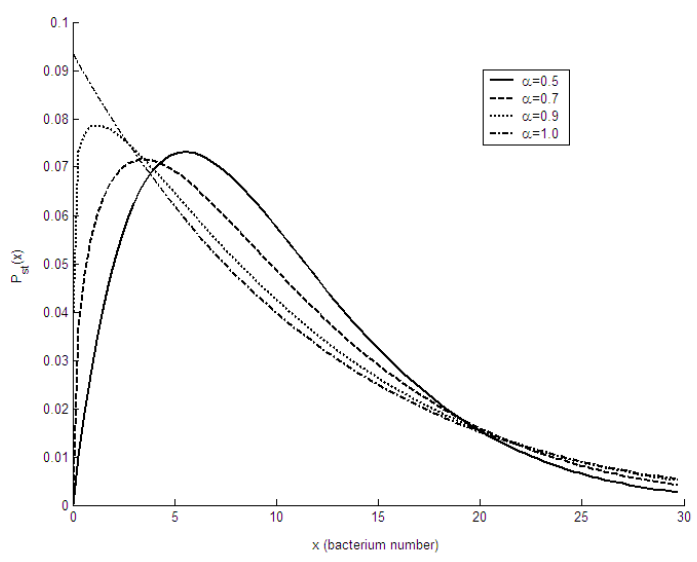

FIG. 2: Plot of $P_{s t}(x)$ (probability density) vs. $x$ (bacterium number) for high values of the noise intensity $a . a=1, b=0.1, \tau=0.1$ and $\alpha=0.5,0.7,0.9$ and 1.0 , respectively(units are arbitrary).

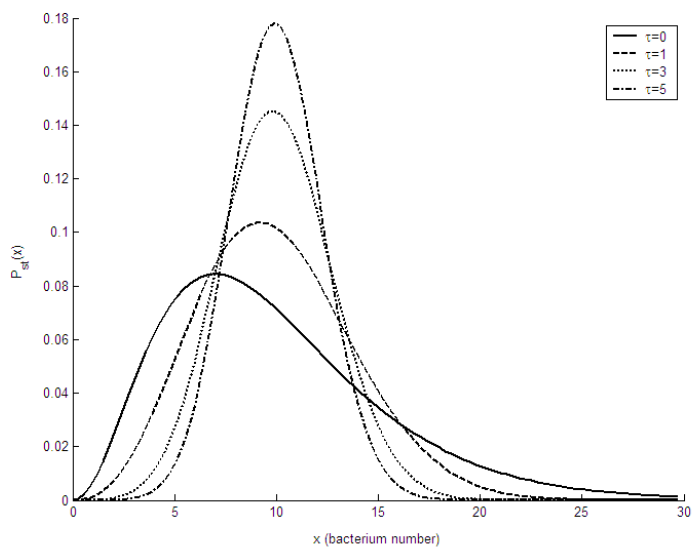

FIG. 3: Plot of $P_{s t}(x)$ (probability density) vs. $x$ (bacterium number) for different values of the noise correlation time $\tau . a=1, b=0.1$, $\alpha=0.3$ and $\tau=0,1,3$ and 5, respectively(units are arbitrary). 


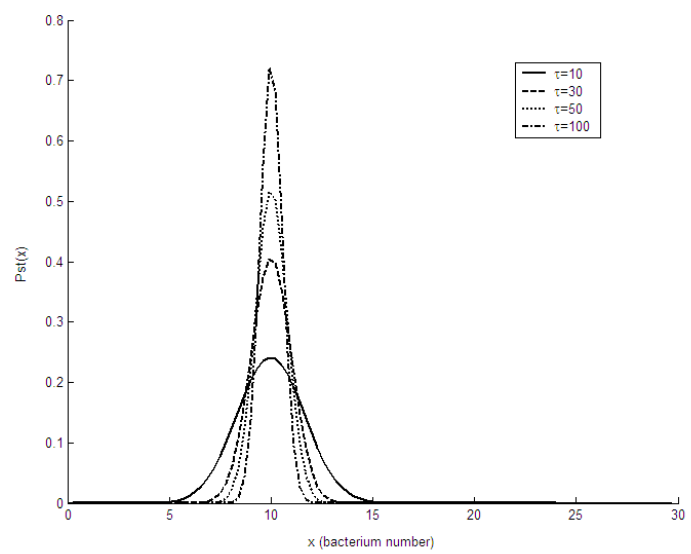

FIG. 4: Plot of $P_{s t}(x)$ (probability density) vs. $x$ (bacterium number) for different values of the noise correlation time $\tau$. $a=1, b=0.1$, $\alpha=0.3$ and $\tau=10,30,50$ and 100, respectively(units are arbitrary).

$$
\begin{gathered}
A(x, \tau)=\frac{a x-b x^{2}}{1+a \tau}+\frac{2 D x^{3}}{(1+a \tau)^{2}}, \\
B(x, \tau)=\frac{D x^{4}}{(1+a \tau)^{2}} .
\end{gathered}
$$

Using the explicit forms of $A(x, \tau)$ and $B(x, \tau)$ we obtain the following SPDF [1213]:

$$
P_{s t}(x)=\frac{N(1+a \tau)^{2}}{D x^{2}} \exp \left[\frac{(2 b x-a)(1+a \tau)}{2 D x^{2}}\right] .
$$

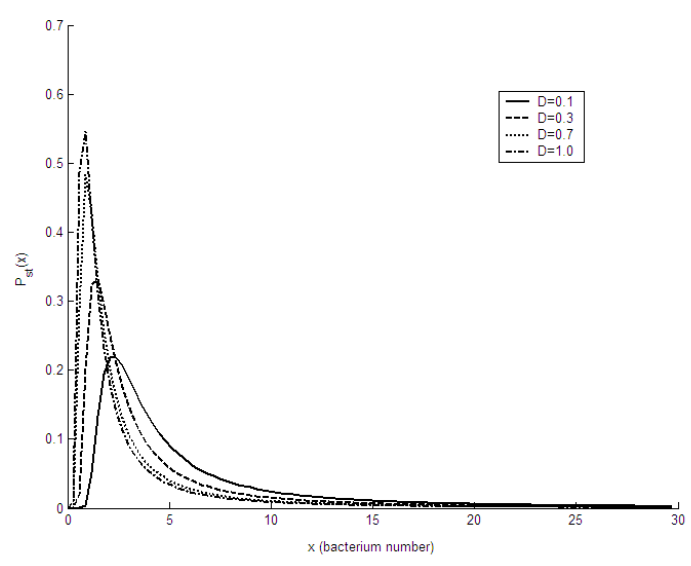

FIG. 5: Plot of $P_{s t}(x)$ (probability density) vs. $x$ (bacterium number) for different values of the multiplicative colored noise intensity $D$. $a=1, b=0.1, \tau=0.1$ and $D=0.1,0.2,0.3$ and 0.4 , respectively(units are arbitrary).

Figure 5 depicts the effects of the strength of the multiplicative colored noise $H(t)$ on the SPDF. We can perceive that the bacterium number is small in the presence of the noise $H(t)$. As $D$ is increased, the maximum value of $P_{s t}(x)$ increases and the peak of SPD moves towards the value of $x=0$. It's clear that the fluctuation of the bacterium decay rate hinders the bacterium growth badly, which means the bacterium number is diminished greatly. Deeply inferred, the fluctuation of the bacterium decay rate caused by the multiplicative colored noise significantly affects the steadiness of the nonlinear biological systems.

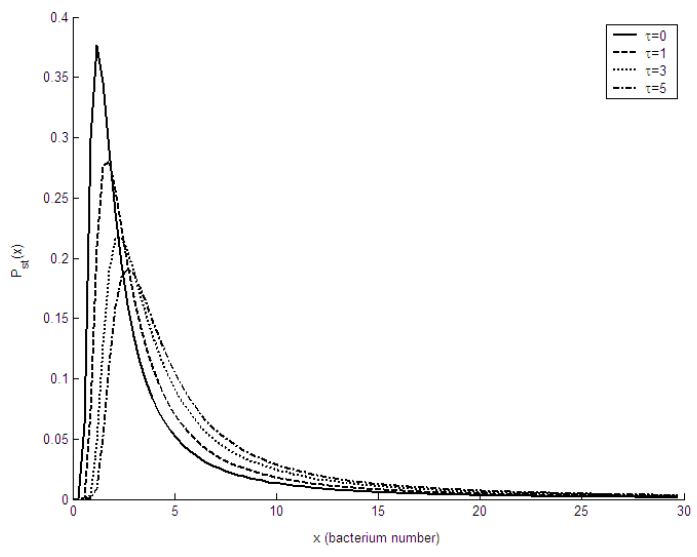

FIG. 6: Plot of $P_{s t}(x)$ (probability density) vs. $x$ (bacterium number) for different values of the noise correlation time $\tau$. $a=1, b=0.1$, $D=0.3$ and $\tau=0,1,3$ and 5 , respectively(units are arbitrary).

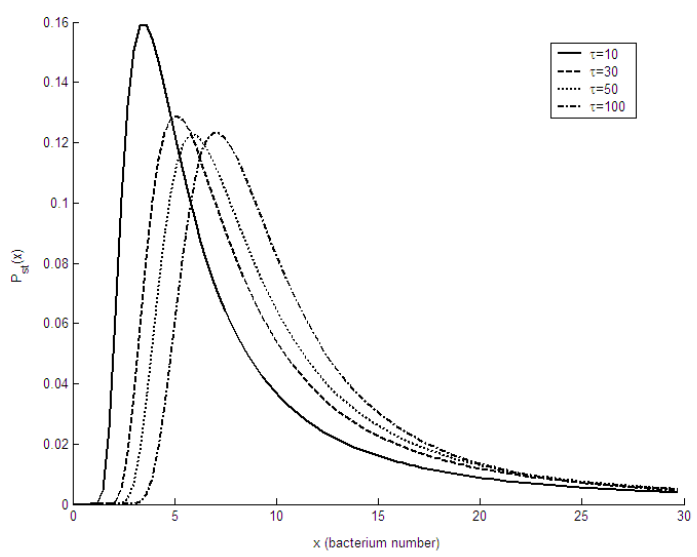

FIG. 7: Plot of $P_{s t}(x)$ (probability density) vs. $x$ (bacterium number) for different values of the noise correlation time $\tau$. $a=1, b=0.1$, $D=0.3$ and $\tau=10,30,50$ and 100, respectively(units are arbitrary).

Figures 6 and 7 display the effects of the correlation time of the multiplicative colored noise $H(t)$ on the SPDF. As $\tau=0$, the colored noise becomes white noise, the position of the extremum of the SPDF is at $x=\frac{1}{3}$. As $\tau$ is increased, the peak of SPD moves towards the larger values of $x$ and the maximum value of $P_{s t}(x)$ decreases. As for the large value of $\tau$, the maximum value of $P_{s t}(x)$ decreases slightly with $\tau$. The bacterium number can increase to about seven as $\tau$ is increased to 100 . 
These indicate that increasing the correlation time of the multiplicative noise results in increased bacteria number. In other words, the large correlation time of the multiplicative colored noise plays a positive role on the evolution of the nonlinear biological systems.

\section{CONCLUSION}

To sum up, we have studied the effects of the external environmental fluctuations on bacterium growth and its steady state properties. Our results demonstrate that the fluctuation of both bacterium growth rate and decay rate caused by the multiplicative colored noise can decrease bacterium number and even make bacterium extinction, which is similar to the case of the white noise. It is inferred that the variation of the external condition can hinder the evolution of the nonlinear biological systems. The multiplicative colored noise makes the maximum of SPD move from a large value of $\mathrm{x}$ to the small values of $\mathrm{x}$, showing that the multiplicative noise is a drift term in the process from the physical point of view. The result is consistent with the one for white noise obtained previously, where we find additive noise is a diffusive factor, while the multiplicative noise is a drift factor in the process of the tumor cell growth [6]. We also find the bacterium number may be increased to some extent by increasing the correlation time of the multiplicative colored noise, while the white noise only hinder the population growth.
[1] A. Fulinski and T. Telejko, Phys. Lett. A 152, 11 (1991).

[2] B. Q. Ai, X.J. Wang, L. G. Liu, M. Nakano, and H. Matsuura, Chin. Phys. Lett. 19, 137 (2002).

[3] Y. Jia and J. R. Li, Phys. Rev. E 53, 5786 (1996).

[4] B. C. Bag, S. K. Banik, and D. S. Ray, Phys. Rev. E 64, 026110 (2001).

[5] B. Q. Ai, X. J. Wang, L. G. Liu, M. Nakano, and H. Matsuura, Commun. Theor. Phys. 37, 25 (2002).

[6] B. Q. Ai, X. J. Wang, G. T. Liu, and L. G. Liu, Phys. Rev. E 67, 022903 (2000).

[7] J. Masoliver, B. J. West, and K. Lindenberg, Phys. Rev. A 35, 3086 (1987)
[8] S. López, M. Prieto, J. Dijkstra, M. S. Dhanoa and J. France, International Journal of Food Microbiology 96 (2004) 289-300.

[9] H. Fujikawa, A. Kai, and S. Morozumi, Food Microbiology, (2004) 501-509.

[10] H. Fujikawa, A. Kai, and S. Morozumi, Journal of the Food Hygienics Society of Japan, (2004) 250-254.

[11] J. C. Panetta, Appl. Math. Lett. 8, 83 (1995).

[12] D. J. Wu, L. Cao, and S. Z. Ke, Phys. Rev. E 50, 2496 (1994).

[13] Table of Integrals, Series and Products, edited by I.S. Gradshteyn and I. M. Ryzhik (Academic, New York, 1980). 